\title{
(C) OPEN ACCESS \\ Confirmed cardiac output on emergency medical services arrival as confounding by indication: an observational study of prehospital airway management in patients with out-of-hospital cardiac arrest
}

\author{
Atsushi Sakurai, ${ }^{\oplus}$ Kosaku Kinoshita, ${ }_{1}^{1}$ Yukihiro Maeda, ${ }^{2}$ Yosuke Homma, ${ }^{\circ}$ \\ Yoshio Tahara, ${ }^{4}$ Naohiro Yonemoto, ${ }^{5}$ Ken Nagao, ${ }^{6}$ Arino Yaguchi, ${ }^{7}$ Naoto Morimura, ${ }^{8}$ \\ SOS-KANTO 2012 Study Group
}

For numbered affiliations see end of article.

\section{Correspondence to}

Dr Atsushi Sakurai, Division of Emergency and Critical Care Medicine Department of Acute Medicine, Nihon University School of Medicine, Tokyo 1738610, Japan;

sakurai.atsushi@nihon-u.ac.jp

Received 4 September 2018

Revised 12 April 2019

Accepted 6 May 2019

Published Online First

6 June 2019

Check for updates

(c) Author(s) (or their employer(s)) 2019. Re-use permitted under CC BY-NC. No commercial re-use. See rights and permissions. Published by BMJ.

To cite: Sakurai A

Kinoshita K, Maeda Y,

et al. Emerg Med J

2019:36:410-415.

\section{ABSTRACT}

Objectives Many registry studies on patients with out-of-hospital cardiac arrest (OHCA) have reported that conventional bag-valve-mask (BVM) ventilation is independently associated with favourable outcomes. This study aimed to compare the data of patients with OCHA with confirmed cardiac output on emergency medical services (EMS) arrival and consider the confounding factors in prehospital airway management studies. Methods This was a cohort study using the registry data for survivors after out-of hospital cardiac arrest in the Kanto region at 2012 in Japan (SOS-KANTO 2012). Survivors who received advanced airway management (AAM) group and a BVM group were compared for confirmed cardiac output on EMS arrival and neurolgical outcome at 1 month. Favourable neurological outcome was defined as a score of one or two on the Cerebral Performance Categories Scale. Multivariable logistic regression was used to adjust the neurological outcome by age, gender, cardiac aetiology, witnessed arrest, shockable rhythm, cardiopulmonary resuscitation performed by a bystander, BVM at prehospital ventilation and presence of confirmed cardiac output on EMS arrival. Results A total of 16452 patients were enrolled in the SOS-KANTO 2012 study, and of those data 12867 were analysed; 5893 patients comprised the AAM group and 6974 comprised the BVM group. Of the study participants, 386 (2.9\%) had confirmed cardiac output on EMS arrival; 340 (2.6\%) of the entire study group had a favourable neurological outcome. The proportion of patients with confirmed cardiac output on EMS arrival was significantly higher in the BVM group (272: 3.9\%) than in the AAM group (114: 1.9\%) ( $95 \% \mathrm{Cl}: 1.65$ to 2.25 ). The proportion of patients with favourable neurological outcomes was 30\% (117/386) in those with cardiac output on EMS arrival compared with $1.8 \%(223 / 12481)$ in those without. The OR for a good neurological outcome with BVM decreased from 3.24 (2.49 to 4.20 ) to 2.60 (1.97 to 3.44) when confirmed cardiac output on EMS arrival was added to the multivariable model analysis.

Conclusion Confirmed cardiac output on EMS arrival should be considered as confounding by indication in observational studies of prehospital airway management.
What is already known on this subject

- Several registry studies on patients with outof-hospital cardiac arrest (OHCA) reported that conventional bag-valve-mask (BVM) ventilation is independently associated with favourable outcome.

- Confounding factors in prehospital airway management studies should be considered in order to identify the best method of managing prehospital airway.

\section{What this study adds}

- Analysing a registry of survivors after OCHA in the Kanto region at 2012 in Japan, we found a significant difference in the proportion of confirmed cardiac output on emergency medical services (EMS) arrival between those who received BVM group compared with those in the advanced airway management group and neurological outcome of patients with confirmed cardiac output on EMS arrival was remarkably good.

- Confounding by indication should be considered in observational studies of prehospital airway management.

\section{INTRODUCTION}

Airway management is one of the most important resuscitation skills and involves the use of several techniques, such as bag-valve-mask (BVM) ventilation, or advanced airway management (AAM) such as supraglottic airway and tracheal intubation. Many registry studies on patients with out-of-hospital cardiac arrest (OHCA) have shown that AAM is independently associated with unfavourable outcome compared with conventional BVM ventilation. Two studies of patients with cardiac aetiology ${ }^{1}$ or with non-traumatic cardiac arrest ${ }^{2}$ found that BVM was associated with survival to discharge. Other studies reported that AAM was 
independently associated with decreased odds of favourable neurological outcome compared with BVM. ${ }^{3}{ }^{4}$ Evans et al showed that AAM was significantly associated with decreased odds of surviving to hospital discharge in traumatic patients and indicated that the relationship between treatment with AAM and poor outcome is biased by the uncontrolled confounding effect of injury severity. ${ }^{5}$ Other confounders were proposed to be the skill of the emergency medical service (EMS) personnel, indications for $\mathrm{AAM}^{6},{ }^{6}$ the timing of insertion of $\mathrm{AAM}^{7}$ and resuscitation time bias. ${ }^{8}$ However, data to confirm whether these factors are confounders have not been reported.

Time interval for whole brain ischaemia has been reported to be critical in determining the outcome of patients with OHCA. ${ }^{910}$ Hence, whether patients have confirmed cardiac output on EMS arrival or not may be related to outcomes. However, whether the patient has confirmed cardiac arrival on arrival of EMS is not in the core data of Utstein variables ${ }^{11} 12$ and has not been investigated as a possible confounder in prior investigations of prehospital cardiac arrest (CA) management.

This study aimed to determine whether patients who have better outcomes and received BVM are more likely to have had cardiac output (and therefore a better prognosis) at the time of EMS arrival.

\section{METHODS}

In the Japanese EMS system, the ambulance crew consists of three EMS members, including at least one emergency lifesaving technician (ELST). ${ }^{13}{ }^{14}$ ELSTs can perform several resuscitative techniques with online medical supervision, including the operation of a semi-automated external defibrillator, insertion of supraglottic airway devices (laryngeal mask airway, laryngeal tube and oesophageal-tracheal twin lumen airway device) and intubation and intravenous administration of epinephrine. In Japan, 'the national protocol of prehospital management of CA for ELSTs' is standard practice. AAM is only indicated in patients with confirmed cardiac or respiratory arrest. ${ }^{15}$ In Japanese prehospital protocol patients with confirmed cardiac output on arrival to EMS are often treated with BVM ventilation.

We conducted a registry study with follow-up of adult $(\geq 18)$ victims of OHCA in the Kanto region (SOS-KANTO) in $2012 .^{13}$ This study was planned and conducted to gather prehospital and inhospital records of patients with CA who were transported to one of the 67 emergency hospitals in the Kanto region of Japan between January 2012 and March 2013. In this study, EMS providers collected the prehospital information in a standardised Utstein style. ${ }^{12}$ In addition, physiological variables present on arrival of the EMS were recorded. These data were signs of cardiac output, spontaneous respiration, pupil diameter and light reflex. Signs of cardiac output was defined as detectable pulse in the internal carotid artery. The SOS-KANTO 2012 study was approved by the relevant institutional review boards of 67 hospitals that participated. The review boards waived the need for written informed consent.

Patients with traumatic CA were included. Those who were transferred from another hospital and those in a persistent vegetative state before CA were excluded from the study. An emergency dispatcher confirmed the CA from a bystander by telephone, based on the 2010 Japan Resuscitation Council Guidelines, ${ }^{16}$ the same as the 2010 International Consensus on Cardiopulmonary Resuscitation and Emergency Cardiovascular Care Science with Treatment Recommendations. ${ }^{17}$

Institutional researchers gathered the data on neurological outcomes of surviving patients within 1 month. Neurological outcomes were evaluated using the Cerebral Performance Categories (CPC) Scale. ${ }^{11}$ Responses were scored as follows: CPC 1, good cerebral performance; CPC 2, moderate cerebral disability; CPC 3, severe cerebral disability; CPC 4, coma/vegetative state and CPC 5, death. Favourable neurological outcome was defined as $\mathrm{CPC} 1$ or $2 . .^{34}$

\section{DATA ANALYSIS}

The cohort was divided into two groups: AAM group (usage of supraglottic airway or intubation) and BVM group. We compared the following data: age, gender, aetiology of CA, witnessed arrest, cardiopulmonary resuscitation (CPR) performed by a bystander, initial cardiac rhythm monitored by EMS providers, and confirmed cardiac output on EMS arrival. Confirmed cardiac output on EMS arrival was defined as a case where the patient had CA at call receipt and confirmed cardiac output (with detectable pulse in the internal carotid artery) immediately on arrival of the EMS. In this study, time interval of CA was not evaluated since the time of return of spontaneous circulation (ROSC) could not be accurately determined in patients with confirmed cardiac output on EMS arrival. Patients who had missing data elements for the period before EMS performed airway management were excluded from the analyses.

Baseline patient characteristics between the two airway management groups were compared using a $\chi^{2}$ test or Fisher's exact test for discrete variables, and a t-test or MannWhitney $U$ test for continuous variables. Neurological outcomes of patients with and without confirmed cardiac output on EMS arrival were compared in the same way. Univariate logistic regression of favourable neurological outcome in patients with OHCA with BVM was achieved. We performed a multivariable logistic regression to calculate the OR and 95\% CI for favourable or poor neurological outcomes at 1 month adjusted by age, gender, cardiac aetiology, witnessed arrest, shockable rhythm, CPR performed by a bystander, BVM at prehospital ventilation and with or without confirmed cardiac output on EMS arrival. These variables were selected a priori, after referring to other studies. ${ }^{1-5}$ Age was fitted as a continuous variable.

The level of significance was set at $p<0.05$. Analyses were performed using SPSS (V.16; SPSS, Chicago, Illinois, USA) statistical software packages.

\section{RESULTS}

A total of $16452(100 \%)$ patients with CA were enrolled in the SOS-KANTO 2012 study, and 3585 (21.8\%) patients were excluded from the analysis; 554 (3.4\%) did not meet the inclusion criteria and the remaining 3031 (18.4\%) had incomplete data for analysis. Therefore, data for this analysis were available in 12867 (78.2\%) patients; 5893 (35.8\%) patients in the AAM group, and 6974 (42.4\%) in the BVM group (figure 1). Of the study participants, there were 386 (2.9\%) patients with confirmed cardiac output on EMS arrival; 340 (2.6\%) of those had favourable neurological outcomes.

The AAM group was significantly $(\mathrm{p}<0.001)$ older at $72.0 \pm 15.8$ (mean \pm SD) than that of BVM $(70.2 \pm 17.5)$ (table 1 and figure 2). There were more patients in AAM with cardiac aetiology, who received CPR by a bystander and who had shockable rhythm on arrival by EMS than the BVM group. The BVM group had a significantly higher incidence of confirmed cardiac output on EMS arrival at $272(3.6 \%)$ compared with the AAM group at $114(1.9 \%)(\mathrm{p}<0.0001)$. The AAM group showed 


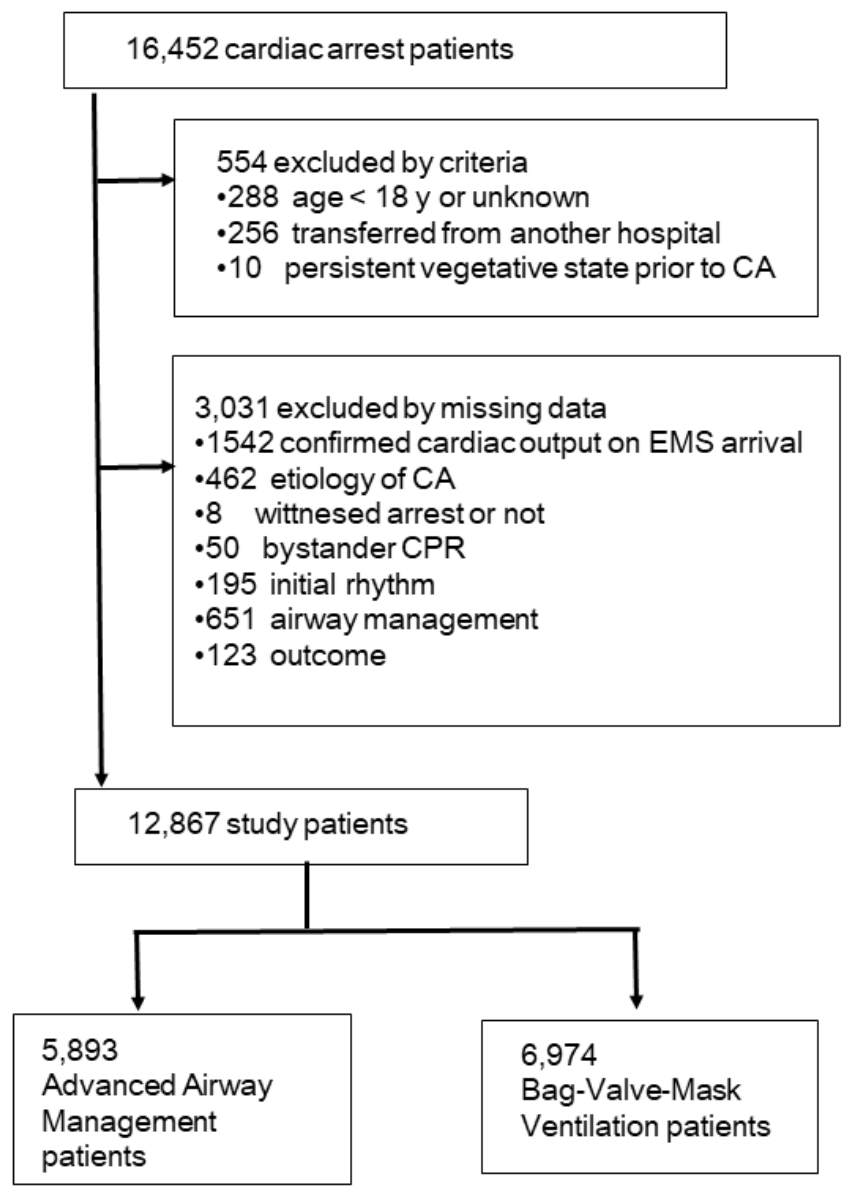

Figure 1 Flow diagram of the study population. CA, cardiac arrest; EMS, emergency medical services; CPR, cardiopulmonary resuscitation.

lower survival rates and poorer neurological outcomes (table 1 and figure 2).

Among patients with confirmed cardiac output on EMS arrival, $30.3 \% \quad(117 / 386)$ had a favourable neurological outcome, whereas neurological outcome was favourable in only $1.8 \%(223 / 12481)$ in those without cardiac output on EMS arrival. Patients with confirmed cardiac output on EMS arrival had considerably higher rates of good neurological outcomes than those without confirmed cardiac output on EMS arrival (table 2).

Univariate logistic regression of good neurological outcome in patients with OHCA who received BVM gave an OR of 2.43 (95\% CI: 1.91 to 3.19 ). In the multivariate model that did not

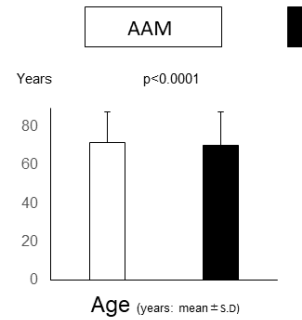

\section{BVM}
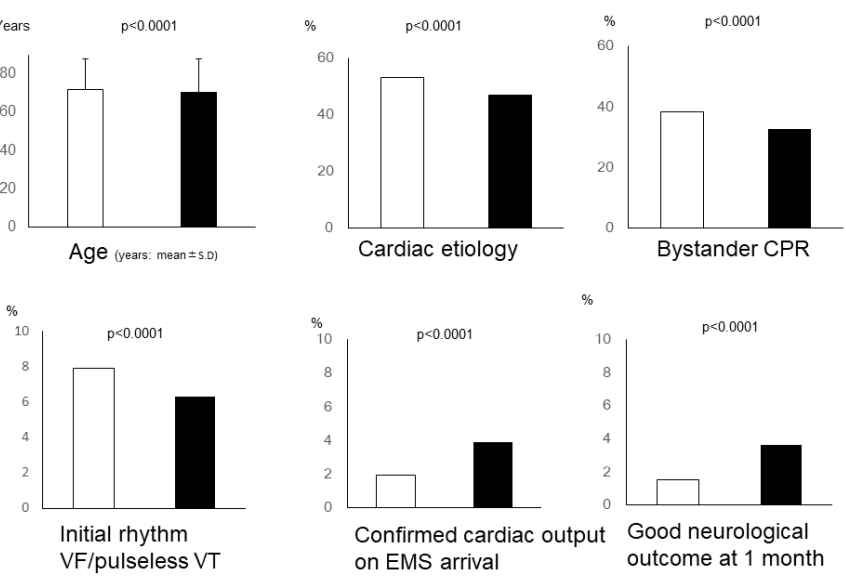

Figure 2 Graphs with elements, which showed significant differences in table 1. AAM, advanced airway management group; BVM, bagvalve-mask ventilation group; CA, cardiac arrest; CPR, cardiopulmonary resuscitation; EMS, emergency medical services; VF, ventricular fibrillation; VT, ventricular tachycardia.

include whether or not cardiac output was present, the OR for good neurological outcome with BVM was 3.24 (2.49 to 4.20), but this was reduced to 2.60 (1.97 to 3.44$)$ when confirmed cardiac output on EMS arrival was added to the multivariable model analysis (table 2).

\section{DISCUSSION}

In this study, patients who received BVM had a better neurological outcomes than those who had AAM. However, the proportion of patients with confirmed cardiac output on EMS arrival was significantly higher in the BVM group than in the AAM group. Neurological outcomes in patients with confirmed cardiac output on EMS arrival was remarkably good and this characteristic had a significant positive association with favourable neurological outcomes in a multivariable logistic regression.

The outcome of patients with OHCA depends on the duration of circulatory arrest. Komatsu et al reported that shortening the time interval from receiving a call until ROSC was the only important independent factor to achieve good neurological outcomes in patients with CA. ${ }^{9}$ Soga et al reported that the short interval from the time of collapse until ROSC was an essential factor to achieve good neurological outcomes in patients with OHCA treated with therapeutic hypothermia. ${ }^{10}$ Patients with confirmed cardiac output on EMS arrival will

Table 1 Data comparison between the advanced airway management group and the bag-valve-mask ventilation group

\begin{tabular}{|c|c|c|c|c|}
\hline & $\begin{array}{l}\text { Advanced airway management } \\
(n=5893)\end{array}$ & $\begin{array}{l}\text { Bag-valve-mask (BVM) ventilation } \\
(n=6974)\end{array}$ & $95 \% \mathrm{Cl}$ & $P$ value \\
\hline Age (years); median (25-75 percentile) & $76(64-84)$ & $74(60-83)$ & 0.99 to 1.00 & $<0.0001$ \\
\hline Gender ( $\%$ male) & $3599(61.0)$ & $4185(60.0)$ & 0.89 to 1.03 & 0.2250 \\
\hline Cardiac aetiology (\%) & $3116(52.9)$ & $3263(46.8)$ & 0.73 to 0.84 & $<0.0001$ \\
\hline Witnessed arrest (\%) & $2889(49.0)$ & $3333(47.8)$ & 0.89 to 1.02 & 0.1670 \\
\hline Bystander CPR (\%) & $2245(38.1)$ & $2269(32.5)$ & 0.73 to 0.84 & $<0.0001$ \\
\hline Initial rhythm VF/pulseless VT (\%) & $468(7.9)$ & $442(6.3)$ & 0.69 to 0.90 & $<0.0001$ \\
\hline Confirmed cardiac output on EMS arrival (\%) & $114(1.9)$ & $272(3.9)$ & 1.65 to 2.57 & $<0.0001$ \\
\hline Good neurological outcome at 1 month (\%) & $89(1.5)$ & $251(3.6)$ & 1.91 to 3.19 & $<0.0001$ \\
\hline
\end{tabular}

CPR, cardiopulmonary resuscitation; EMS, emergency medical services; VF, ventricular fibrillation; VT, ventricular tachycardia. 


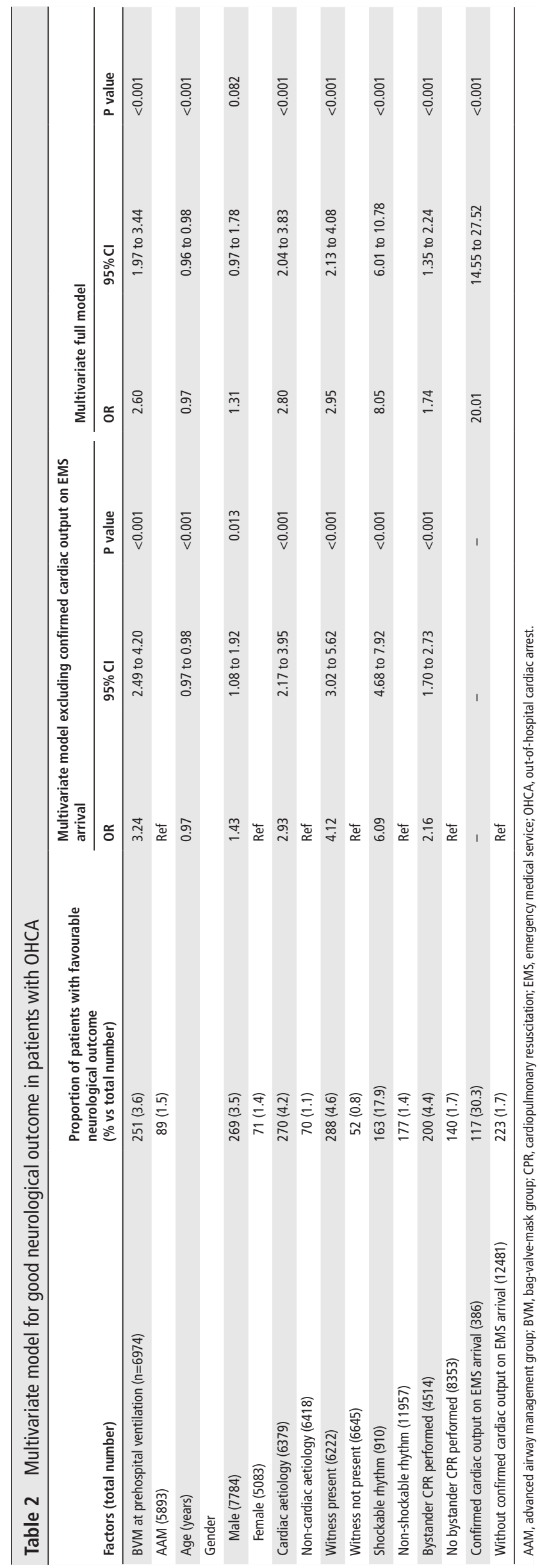

more likely experience a shorter duration of arrest, and it may be anticipated that these patients may consequently have improved neurological outcomes. In this study, the neurological outcomes of patients with confirmed cardiac output on EMS arrival was remarkably good and independently associated with a favourable neurological outcome, with a significant OR. Furthermore, when confirmed cardiac output on EMS arrival was accounted for in the logistic regression, the association between AAM and poorer neurological outcome decreased. This may explain prior findings on the association of AAM and poorer outcome. ${ }^{1-5}$ It would not be surprising that, if patients already have confirmed cardiac output on arrival of EMS, intubation might be withheld. This suggests that confirmed cardiac output on EMS arrival should be a potential confounder.

It is of interest that this finding has not been reported in prior studies. In all registry studies ${ }^{1-5}$ in which AAM was independently associated with unfavourable outcomes, outcomes were adjusted for age, aetiology, CPR by an initial bystander rhythm, rhythm on EMS arrival and other confounding factors. However, no study has considered the presence of cardiac output. Our findings suggest that confirmed cardiac output on EMS arrival could be considered as an unmeasured confounder in these prior studies, going forward, confirmed cardiac output on EMS arrival should be recorded in registries and included in studies to help determine the best method of managing the airway of patients who experience OHCA. Adding it to the core data reported using the Utstein style may also be important.

Izawa et al reported that AAM was associated with survival only among patients with non-shockable rhythms, and suggested that the reason that their results differed from that of the study by Hasegawa et al was due to resuscitation time bias. ${ }^{8}$ However, they excluded patients with missing data in time-dependent variables, including ROSC. Usually, ROSC time with confirmed cardiac output on EMS arrival may be undetermined, and many patients with this characteristic might have been excluded by this criterion in the study by Izawa et al. This suggests that we should consider confirmed cardiac output on EMS arrival as a clear confounder and should perform analyses with this element in a prehospital study.

This study has several limitations. A considerable number of patients were excluded due to missing values in the registration study. It is likely that outcome was influenced by other unrecorded variables.

In this study, duration of CA was not evaluated since time of ROSC could not be accurately determined in patients with confirmed cardiac output on EMS arrival. ROSC may occur at some time between the emergency call and the arrival of the ambulance team, but most bystanders are laypersons and are unable to determine the time of ROSC accurately. The method used in confirming that all patients with confirmed cardiac output on EMS arrival actually experienced CA is another concern. Emergency dispatchers diagnosed CA based on the guidelines; however, this depended on information from a layperson and by telephone. Bahr et al reported that laypersons have poor skills in checking the carotid pulse. ${ }^{18} \mathrm{~A}$ dispatcher cannot accurately distinguish whether the patient experienced $\mathrm{CA}$ or not at call receipt and there is no standard method of recording this in registry studies. These patients would nevertheless have been placed in a registry. This limitation exists in all prior OHCA studies. We suggest that confirmed cardiac output on EMS arrival should be at least recorded and evaluated with the possibility that a significant proportion of these patients may never have been in CA at all and would, consequently, have been 
expected to have better neurological outcomes than even those with the shortest CA interval.

\section{CONCLUSION}

Presence or absence confirmed cardiac output on EMS arrival can introduce bias into OHCA studies, potentially leading to confounding by indication on the choice of airway management. This should be considered when evaluating the effectiveness of prehospital airway management.

\author{
Author affiliations \\ 'Division of Emergency and Critical Care Medicine Department of Acute Medicine, \\ Nihon University School of Medicine, Itabashi, Japan \\ ${ }^{2}$ Department of Health Care Services Management, Nihon University School of \\ Medicine, Itabashi, Japan \\ ${ }^{3}$ Emergency Medicine, Tokyo Bay Urayasu Ichikawa Medical Center, Urayasu, Japan \\ ${ }^{4}$ National Cerebral and Cardiovascular Center Hospital, Suita, Japan \\ ${ }^{5}$ Department of Public Health, Kyoto University Graduate School of Medicine, Kyoto, \\ Japan \\ ${ }^{6}$ Cardiovascular Center, Nihon University Hospital, Chiyoda, Japan \\ ${ }^{7}$ Department of Critical Care and Emergency Medicine, Tokyo Women's Medical \\ University, Shinjuku, Japan \\ ${ }^{8}$ Department of Acute Medicine, The University of Tokyo Graduate School of \\ Medicine, Bunkyo, Japan
}

Acknowledgements The authors would like to thank Ms. Mai Matsumoto and the secretariat members of the Japanese Association for Acute Medicine of Kanto who assisted in the collection of data and in the coordinating of this project. This study was carried out with the support of the Japanese Association for Acute Medicine of Kanto.

Collaborators SOS-KANTO 2012 Study Group: Tokai University School of Medicine (Sadaki Inokuchi MD); St. Marianna University School of Medicine, Yokohama Seibu Hospital (Yoshihiro Masui MD); Koto Hospital (Kunihisa Miura MD); Saitama Medical Center Advanced Tertiary Medical Center (Haruhiko Tsutsumi MD); Kawasaki Municipal Hospital Emergency and Critical Care Center (Kiyotsugu Takuma MD); Yokohama Municipal Citizen's Hospital (Ishihara Atsushi MD); Japanese Red Cross Maebashi Hospital (Minoru Nakano MD); Juntendo University Urayasu Hospital (Hiroshi Tanaka MD); Dokkyo Medical University Koshigaya Hospital (Keiichi Ikegami MD); Hachioji Medical Center of Tokyo Medical University (Takao Arai MD); Tokyo Women's Medical University Hospital (Arino Yaguchi MD); Kimitsu Chuo Hospital (Nobuya Kitamura MD); Chiba University Graduate School of Medicine (Shigeto Oda MD); Saiseikai Utsunomiya Hospital (Kenji Kobayashi MD); Mito Saiseikai General Hospital (Takayuki Suda MD); Dokkyo Medical University (Kazuyuki Ono MD); Yokohama City University Medical Center (Naoto Morimura MD); National Hospital Organization Yokohama Medical Center (Ryosuke Furuya MD); National Disaster Medical Center (Yuichi Koido MD); Yamanashi Prefectural Central Hospital (Fumiaki Iwase MD); Surugadai Nihon University Hospital (Ken Nagao MD); Yokohama Rosai Hospital (Shigeru Kanesaka MD); Showa General Hospital (Yasusei Okada MD); Nippon Medical School Tamanagayama Hospital (Kyoko Unemoto MD); Tokyo Women's Medical University Yachiyo Medical Center (Tomohito Sadahiro MD); Awa Regional Medical Center (Masayuki lyanaga MD); Todachuo General Hospital (Asaki Muraoka MD); Japanese Red Cross Medical Center (Munehiro Hayashi MD); St. Luke's International Hospital (Shinichi Ishimatsu MD); Showa University School of Medicine (Yasufumi Miyake MD); Totsuka Kyoritsu Hospital 1 (Hideo Yokokawa MD); St. Marianna University School of Medicine (Yasuaki Koyama MD); National Hospital Organization Mito Medical Center (Asuka Tsuchiya MD); Tokyo Metropolitan Tama Medical Center (Tetsuya Kashiyama MD); Showa University Fujigaoka Hospital (Munetaka Hayashi MD); Gunma University Graduate School of Medicine (Kiyohiro Oshima MD); Saitama Red Cross Hospital (Kazuya Kiyota MD); Tokyo Metropolitan Bokutoh Hospital (Yuichi Hamabe MD); Nippon Medical School Hospital (Hiroyuki Yokota MD); Keio University Hospital (Shingo Hori MD); Chiba Emergency Medical Center (Shin Inaba MD); Teikyo University School of Medicine (Tetsuya Sakamoto MD); Japanese Red Cross Musashino Hospital (Naoshige Harada MD); National Center for Global Health and Medicine Hospital (Akio Kimura MD); Tokyo Metropolitan Police Hospital (Masayuki Kanai MD); Medical Hospital of Tokyo Medical and Dental University (Yasuhiro Otomo MD); Juntendo University Nerima Hospital (Manabu Sugita MD); Nihon University School of Medicine (Kosaku Kinoshita MD); Toho University Ohashi Medical Center (Takatoshi Sakurai MD); Saiseikai Yokohamashi Tobu Hospital (Mitsuhide Kitano MD); Nippon Medical School Musashikosugi Hospital (Kiyoshi Matsuda MD); Tokyo Rosai Hospital (Kotaro Tanaka MD); Toho University Omori Medical Center (Katsunori Yoshihara MD); Hiratsuka City Hospital (Kikuo Yoh MD); Yokosuka Kyosai Hospital (Junichi Suzuki MD); Saiseikai Yokohamashi Nambu Hospital (Hiroshi Toyoda MD); Nippon Medical School Chiba Hokusoh Hospital (Kunihiro Mashiko MD); Tokyo Metropolitan Children's Medical
Centre (Naoki Shimizu MD); National Medical Center for Children and Mothers (Takashi Muguruma MD); Chiba Aoba Municipal Hospital (Tadanaga Shimada MD); Kuki General Hospital (Yoshiro Kobe MD); Matsudo City Hospital (Tomohisa Shoko MD); Japanese Red Cross Narita Hospital (Kazuya Nakanishi MD); Tokyo Bay Urayasu/lchikawa Medical Center (Takashi Shiga MD); NTT Medical Center Tokyo (Takefumi Yamamoto MD); Tokyo Saiseikai Central Hospital (Kazuhiko Sekine MD); Fuji Heavy Industries Health Insurance Society OTA Memorial Hospital (Shinichi Izuka MD). http://www.jaam-kanto.jp/sos_kanto/sos_kanto2012_contributors.html

Contributors AS contributed to study conception and design, acquisition of data, interpretation of data and critical revision of the manuscript for important intellectual content, and provided intellectual input to the research and manuscript. KK, YM and NY contributed to study conception and design, acquisition of data, analysis and interpretation of data, statistical analysis, drafting of the manuscript and critical revision of the manuscript for important intellectual content, and provided intellectual input to the research and manuscript. YH, YT, KN, AY and NM contributed to acquisition of data and critical revision of the manuscript for important intellectual content, and provided intellectual input to the research and manuscript. All authors read and approved the manuscript.

Funding The authors have not declared a specific grant for this research from any funding agency in the public, commercial or not-for-profit sectors.

Competing interests None declared.

\section{Patient consent for publication Not required.}

Ethics approval The SOS-KANTO 2012 study was approved by the relevant institutional review boards of 67 hospitals that participated.

Provenance and peer review Not commissioned; externally peer reviewed.

Open access This is an open access article distributed in accordance with the Creative Commons Attribution Non Commercial (CC BY-NC 4.0) license, which permits others to distribute, remix, adapt, build upon this work non-commercially, and license their derivative works on different terms, provided the original work is properly cited, appropriate credit is given, any changes made indicated, and the use is non-commercial. See: http://creativecommons.org/licenses/by-nc/4.0/.

\section{REFERENCES}

1 Shin SD, Ahn KO, Song KJ, et al. Out-of-hospital airway management and cardiac arrest outcomes: a propensity score matched analysis. Resuscitation 2012;83:313-9.

2 Hanif MA, Kaji AH, Niemann JT. Advanced airway management does not improve outcome of out-of-hospital cardiac arrest. Acad Emerg Med 2010;17:926-31.

3 Hasegawa K, Hiraide A, Chang Y, et al. Association of prehospital advanced airway management with neurologic outcome and survival in patients with out-of-hospital cardiac arrest. JAMA 2013;309:257-66.

4 McMullan J, Gerecht R, Bonomo J, et al. Airway management and out-of-hospital cardiac arrest outcome in the CARES registry. Resuscitation 2014;85:617-22.

5 Evans CC, Petersen A, Meier EN, et al. Prehospital traumatic cardiac arrest: Management and outcomes from the resuscitation outcomes consortium epistrytrauma and PROPHET registries. J Trauma Acute Care Surg 2016;81:285-93.

6 Norii T, Hatanaka T. Prehospital airway management for out-of-hospital cardiac arrest. JAMA 2013;309:1888-9.

7 Benoit JL, Prince DK, Wang HE. Mechanisms linking advanced airway management and cardiac arrest outcomes. Resuscitation 2015;93:124-7.

8 Izawa J, Komukai S, Gibo K, et al. Pre-hospital advanced airway management for adults with out-of-hospital cardiac arrest: nationwide cohort study. BMJ 2019:364:1430.

9 Komatsu T, Kinoshita K, Sakurai A, et al. Shorter time until return of spontaneous circulation is the only independent factor for a good neurological outcome in patients with postcardiac arrest syndrome. Emerg Med J 2014;31:549-55.

10 Soga T, Nagao K, Sawano H, et al. Neurological benefit of therapeutic hypothermia following return of spontaneous circulation for out-of-hospital non-shockable cardiac arrest. Circ J 2012;76:2579-85.

11 Cummins RO, Chamberlain DA, Abramson NS, et al. Recommended guidelines for uniform reporting of data from out-of-hospital cardiac arrest: the Utstein Style. A statement for health professionals from a task force of the American Heart Association, the European Resuscitation Council, the Heart and Stroke Foundation of Canada, and the Australian Resuscitation Council. Circulation 1991;84:960-75.

12 Perkins GD, Jacobs IG, Nadkarni VM, et al. Cardiac Arrest and Cardiopulmonary Resuscitation Outcome Reports: Update of the Utstein Resuscitation Registry Templates for Out-of-Hospital Cardiac Arrest: A Statement for Healthcare Professionals From a Task Force of the International Liaison Committee on Resuscitation (American Heart Association, European Resuscitation Council, Australian and New Zealand Council on Resuscitation, Heart and Stroke Foundation of Canada, InterAmerican Heart Foundation, Resuscitation Council of Southern Africa, Resuscitation Council of Asia); and the American Heart Association Emergency Cardiovascular Care Committee and the Council on Cardiopulmonary, Critical Care, Perioperative and Resuscitation. Resuscitation 2015;96:328-40. 
13 SOS-KANTO 2012 Study Group. Changes in pre- and in-hospital management and outcomes for out-of-hospital cardiac arrest between 2002 and 2012 in Kanto, Japan: the SOS-KANTO 2012 Study. Acute Med Surg 2015;2:225-33.

14 Kashiura M, Hamabe Y, Akashi A, et al. Association between cardiopulmonary resuscitation duration and one-month neurological outcomes for out-of-hospital cardiac arrest: a prospective cohort study. BMC Anesthesiol 2017;17:59.

15 SOS-KANTO study group. Comparison of arterial blood gases of laryngeal mask airway and bag-valve-mask ventilation in out-of-hospital cardiac arrests. Circ J 2009;73:490-6.
16 Tanigawa K, Ishikawa M, Takeuchi A, et al. Basic life support: Japan resuscitation council Guideline 2010. Tokyo: Herusu shuppan, 2010:16-43.

17 Sayre MR, Koster RW, Botha M, et al. Part 5: adult basic life support: 2010 International Consensus on Cardiopulmonary Resuscitation and Emergency Cardiovascular Care Science With Treatment Recommendations. Circulation 2010;122:\$298-324.

18 Bahr J, Klingler H, Panzer W, et al. Skills of lay people in checking the carotid pulse. Resuscitation 1997;35:23-6. 